\title{
Epigenetic Regulation of Hematopoietic Stem Cells
}

\author{
Shilpa Sharma, Gangenahalli Gurudutta \\ Division of Stem Cell Gene Therapy Research, Institute of Nuclear Medicine E Allied Sciences (INMAS), Delhi, India
}

Hematopoietic stem cells are endowed with a distinct potential to bolster self-renewal and to generate progeny that differentiate into mature cells of myeloid and lymphoid lineages. Both hematopoietic stem cells and mature cells have the same genome, but their gene expression is controlled by an additional layer of epigenetics such as DNA methylation and post-translational histone modifications, enabling each cell-type to acquire various forms and functions. Until recently, several studies have largely focussed on the transcription factors andniche factors for the understanding of the molecular mechanisms by which hematopoietic cells replicate and differentiate. Several lines of emerging evidence suggest that epigenetic modifications eventually result in a defined chromatin structure and an "individual" gene expression pattern, which play an essential role in the regulation of hematopoietic stem cell self-renewal and differentiation. Distinct epigenetic marks decide which sets of genes may be expressed and which genes are kept silent. Epigenetic mechanisms are interdependent and ensure lifelong production of blood and bone marrow, thereby contributing to stem cell homeostasis. The epigenetic analysis of hematopoiesis raises the exciting possibility that chromatin structure is dynamic enough for regulated expression of genes. Though controlled chromatin accessibility plays an essential role in maintaining blood homeostasis; mutations in chromatin impacts on the regulation of genes critical to the development of leukemia. In this review, we explored the contribution of epigenetic machinery which has implications for the ramification of molecular details of hematopoietic self-renewal for normal development and underlying events that potentially co-operate to induce leukemia.

Keywords: Epigenetics, Self-renewal, Regulation, Hematopoietic stem cells

\section{Introduction}

Hematopoietic stem and progenitor cells (HSPCs) possess the capacity for self-renewal (mitotic division leading to production of the same stem cells without loss of developmental potential) and differentiation (asymmetric division leading to unequal production of daughter cells from

Accepted for publication December 28, 2015, Published online May 30, 2016 Correspondence to Gangenahalli Gurudutta

Division of Stem Cell Gene Therapy Research, Institute of Nuclear Medicine \& Allied Sciences (INMAS), Lucknow Road, Timarpur, Delhi 110054, India

Tel: +91-11-23905144, Fax: +91-11-25737049

E-mail: gugdutta@rediffmail.com

(a) This is an open-access article distributed under the terms of the Creative Commons Attribution Non-Commercial License (http://creativecommons.org/ licenses/by-nc/4.0/), which permits unrestricted non-commercial use, distribution, and reproduction in any medium, provided the original work is properly cited. the same mother cells). Though these two opposing forces of self-renewal and differentiation act on HSPCs, hematopoietic homeostasis is maintained throughout the lifetime of an individual by carefully orchestrated sequence specific transcription factor networks, genes and signaling pathways $(1,2)$. Transcription factors recruit cofactors to gene regulatory regions, which facilitate or inhibit gene transcription by chromatin modification (3). Modulation of gene expression by chromatin modification on global and gene-specific level is 'Epigenetic' regulation. Since the hematopoietic cell lineage development follows a strict hierarchical pattern emanating from a primary single HSC, the hematopoietic system provides an ideal model system to study the correlation between epigenetically directed changes in chromatin structure and gradual restricted cell potential during differentiation. DNA organized in loose chromatin (euchromatin) is readily available for gene expression and contribute for the maintenance of pluripotency. 
Conversely, DNA tightly packed into dense chromatin (heterochromatin) has genetic code buried effectively and become inconspicuous for genetic reading and transcription. Epigenetic reprogramming describes stable and inheritable alterations in gene expression that occur without permanent changes in DNA sequence. Such changes include active or repressive histone modifications working in closed concert with DNA methylation (2). Major covalent modifications that occur at histone tails are acetylation/deacetylation by histone acetyltransferases/histone deacetylases and methylation/demethylation by histone methyltransferases/histone demethylases. Methylated histone arginine and lysine residues are considered static modifications because of low levels of methylgroup turnover in chromatin. With the identification of enzymes that counteract histone methylation, dynamic nature of these modifications has emerged (Fig. 1) (4). In contrast to a phenomenal variety of histone modifications, DNA methylation is simply binding of a methyl group at carbon-5 position of cytosine base by DNA methyltransferases (DNMTs), primarily in cytosine-phosphate-guanine $(\mathrm{CpG})$ dinucleotides or $\mathrm{CpG}$ islands (5). DNA methylation leads to stable long-term repression. While histone methylation events may be associated with gene activation, certain forms of histone methylation cause a local formation of heterochromatin, which is readily reversible. Apparently, DNA methylation and histonemodifications are dependent on one another, their cross-talk is mediated by biochemical interactions between
SET histone methyltransferases and DNA methyltransferases (6). The demethylation process is quite complex and involves proteins, such as the TET (ten-eleven translocation) family of proteins. TET1 was found to catalyze the oxidation of 5-methylcytosine, a well-known epigenetic mark, into 5-hydroxymethylcytosine in mammalian DNA (7). Interestingly, regulation of promoter demethylation-associated 463 genes is more frequent in the hematopoietic system than in non-hematopoietic tissues (241 genes), supporting the overall influence of demethylation signals in driving the tight time-and step-controlled hematopoietic cell fate determination. For example, promoter methylation of hematopoietic genes is linked to stemness as demethylation occur during early embryonic development and later on during hematopoietic differentiation. Genome wide methylation status of induced pluripotent stem cells (iPSCs) and somatic CD34 ${ }^{+}$cells further confirm the role of DNA methylation in the regulation of hematopoietic gene expression. DNA methylation functions during iPS cell generation by silencing of genes important in differentiation, and facilitating chromatin remodelling. Conversely, DNA demethylation appears to play an important role in reactivating pluripotency genes, particularly in the late stages of the reprogramming process. Repressive epigenetic modifications, (H3K9me3 and DNA methylation) at the promoter regions, occasionally observed in partially reprogrammed iPS cells, fails to produce the robust reactivation of pluripotency genes (8). The acetylation of

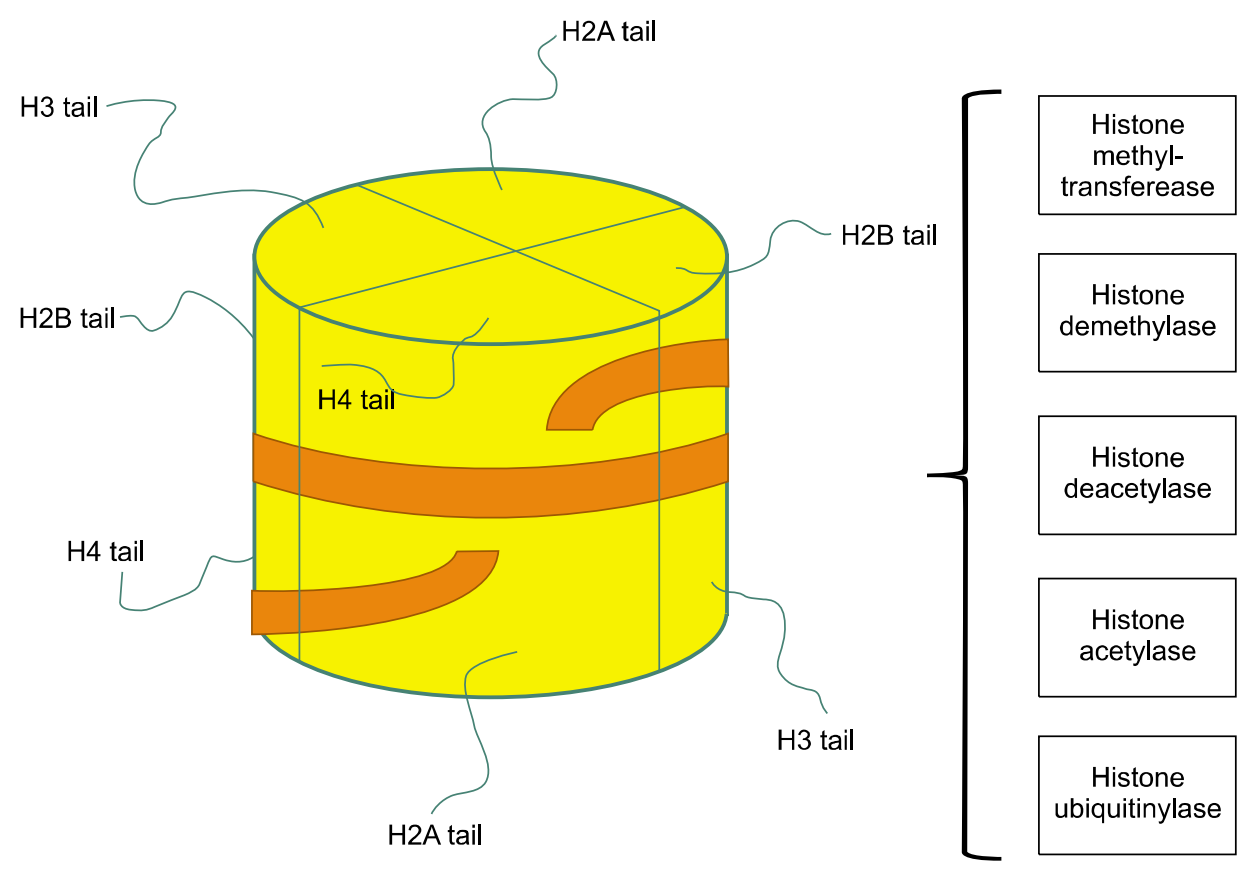

Fig. 1. Schematic of nucleosome structure. Nucleosomes are the smallest structural unit of chromatin. Nucleosome consists of two copies of each core histone $(\mathrm{H} 2 \mathrm{~A}, \mathrm{H} 2 \mathrm{~B}, \mathrm{H} 3$ and $\mathrm{H} 4$ ) and $\sim 150$ bp DNA. The N-terminal tail of each histone is extruded from the nucleosome. Amino acids in histone tails can be modified by numerous enzymes bringing acetylation, methylation, phosphorylation, ubiquitination and other substitutions, creating a complex 'bar' code, which may influence chromatin structure by affecting histone-histone and histone-DNA interactions. Post-translational modifications of histones regulate transcription of genes important for self-renewal and dif-

Nucleosome core ferentiation. 
histones formthe relaxed chromatin, conducive to gene expression activation. The primary site of histone acetylation is the addition of acetyl moiety to the $\epsilon$-amino terminal of lysine residues, which change the physicochemical properties of histone proteins, interfering with the electrostatic attraction between positively charged histones and negatively charged DNA. In contrast, the removal of acetyl groups from the acetylated histones, "histone deacetylation", forms the basis of inactive chromatin environment (heterochromatin) (2). Stem cells in the primitive and differentiated stage organize their level by multitude layers of molecular epigenetic events to impose flexible but precise control to establish important regulatory gene expression patterns. In this review, we highlight recent findings in epigenetic regulation in hematopoietic stem cells and their progeny. We also delineated the mechanism of aberrant placement of epigenetic marks and mutations in the epigenetic machinery involved in hematopoietic malignancies.

\section{Epigenetic events from self-renewal to differentiation}

\section{DNA methylation}

Stem/progenitor cell differentiation is linked to gradual methylation primarily in cytosine-phosphate-guanine $(\mathrm{CpG})$ dinucleotides or $\mathrm{CpG}$ islands that reside within DNA regulatory sequence by DNA methyltransferases. However, whether DNA methylation induces transcriptional silencing or preserves silence state of chromatin, remains enigmatic (2). DNMT3a and DNMT3b showcomplementary de novo methylation patternsresponsible for silencing of self-renewal genes in HSCs (9). Consistent with this,loss of DNMT3a results in expansion of HSC population by impeding differentiation and upregulation of self-renewal genes (Runxl and Gata3). Combined loss of DNMT3a and DNMT3b in HSCs result in enhancement of HSC self-renewal by activating $\beta$-catenin signalling (10). Methylation is maintained by DNMT1 to permit efficient hematopoietic differentiation. DNMT1 is crucial for the progression of stem cells to multipotent progenitors to lineage-restricted myeloid progenitors and regulating cell cycle entry (11). DNA methylation is known to interplay with other chromatin marks, such as histone modifications (12). Extrinsic factors also effect epigenetic regulation. The dynamic interplay between the epigenetic changes and gender-specific hormone apolipoprotein E (Apoe) provide insights for the modulation of a reconstituting potential of HSPCs. In a study, female mice transplanted with truncated DNA methyltransferase 3B isoform, DNMT3B7, resulted in very high expression levels of Apoe. The CpGisland control- ling Apoe expression had lower levels of modified cytosines in DNMT3B7 transgenic HSPCs. DNMT3B7 expression down-regulate hematopoietic number within the female hormonal microenvironment (13). Stem cell protein, SALL4, recruit DNMTs to silence genesto governstem cell self-renewal. It has been investigated that DNMTs and histone deacetylase repressors interact synergistically to reverse the transcriptional repression effect of SALL4 (14). Overexpression of SALL4 leads to increased methylation of silenced genes (H3K4me3 and H3K79me2) in primary HSPCs. During normal hematopoiesis and leukemogenesis, SALL4 mediated expression up-regulates multiple regulatory genes including HOXB4, Notch1, Runx1, Meis1 and Nf-ya, influencing particularly, three important self-renewal pathways involving Bmil, $\beta$-catenin and PTEN. As SALL4 regulate apoptotic pathways both in normal HSCs and leukemic stem cells, Gao et al., deciphered that targeting SALL4 combined with BCL2 antagonist (ABT-737), could lead to leukemic stem cell-specific apoptosis (15). The precise combination of genes which on activation/repression control the processes of driving proliferation and suppressing differentiation, have yet to be defined.

Some genes mutated in cancer recruit histone modifying enzymes and thus alter gene expression. However, the radix of the aberrant methylation of target genes in the tumorous cell is not fully elucidated. Truncated DNMT3B proteins are expressed in primary acute leukemias (2). The PML-RAR gene translocation and RUNX1 in acute promyelocyticleukemia recruits DNMTs to target promoters that change the active chromatin structure to silence status and contributes to its leukemic transformation (16). Recently, hypomethylating agentsemerged as a standard for treatment in myelodysplastic syndrome as they reprogram "methylome" and re-establish hematopoiesis (17).

\section{Histone acetylation}

Protein acetylation regulates HSC self-renewal, proliferation, and their differentiation into committed hematopoietic progenitors. Histone acetyltransferases (HATs) acetylate histone proteins by transferring the acetyl group from acetyl-CoA to specific lysine residues, resulting in a dispersed structure of chromatin, accessible for transcriptional factors. HATs contain p300/CBP (CBP and p300), MYST (Tip60, MOZ, MORF, HBO1 and HMOF), and GNAT (PCAF, Gnc5 and ELP3) family members to regulate normal and malignant hematopoiesis. Multiple chromatin factor complexes, such as NuA4/P300/CBP/HBO1 are required for normal hematopoiesis (17). CBP/p300 regulates self-renewal and differentiation in adult HSCs 
through regulating Gfilb, monocyticleukemia zinc finger protein $(\mathrm{MOZ})$ maintains the generation and development of HSCs and promote proliferation of progenitors by repressing the transcription of p16. It has been proposed that the fusion proteins produced upon translocation of the human $\mathrm{MOZ}$ locus with $\mathrm{CBP} / \mathrm{p} 300$, support the development of leukemia by altering the regulation of $\mathrm{MOZ}$ transcriptional targets (18).

Histone deacetylase inhibitors result in chromatin remodeling to modulate re-expression of silenced tumor suppressor genes in leukemic stem and progenitor cells which,in turn, result in cellular differentiation, inhibition of their proliferation and self-renewal properties (19). Histone deacetylase inhibitor, valproic acid (VPA) increases self-renewal of HSCs by blocking differentiation at multiple regulatory pathways. Epigenetically reprogrammed $\mathrm{CD} 34^{+}$cells on treatment with VPA generate greater numbers of functional stem cells and multilineagehematopoiesis with capacity for bone marrow reconstitution before clinical transplantation. Mechanistically, VPA progress to cell cycle by down-regulating p21, activating Hoxb4 and wntsignaling pathway (20). Walasek et al. showed synergistic effects of VPA and lithium to induce self-renewal by blocking differentiation genes and preserve expression of stem cell-related genes (21). VPA inhibits proliferation and induces apoptosis in leukemic cells by increasing expression of death receptors and their ligands in acute promyelocyticleukemiaand AML-ETO dependent leukemia. Aberrant formation of fusion proteins through chromosomal translocations of HAT and HAT-related genes (e.g. MOZ, MORF, CBP and p300) occurs in leukemia. Mistargeting of such deleterious fusion proteins contributes to global alterations in histone acetylation patterns in cancer. Specific epigenetic gene silencing can occur by aberrant targeting of histone deacetylases to the gene promoter which causes histone hypoacetylation (22).

\section{Histone methylation}

Epigenetic cross-talk between DNA methylation by DNA methyltransferases and histone methylation by histone methyltransferases have ramifications for the understanding of gene transcription for normal development and aberrant gene silencing in tumors. It is not yet clear whether DNA methylation patterns guide histone modifications (histone acetylation and methylation) during gene silencing/or DNA methylation take its cues primarily from histone modification status (23). Histone methylation is a reversible process: methyl marks are established by histone methyltransferases, so-called "writers," and removed by histone demethylating enzymes, termed "eraser".
Three methylation sites on histones are implicated in activation of transcription: H3K4, H3K36, and H3K79. Two of these, H3K4me and H3K36me, have been implicated in transcriptional elongation. Three lysine methylation sites are connected to transcriptional repression: $\mathrm{H} 3 \mathrm{~K} 9$, $\mathrm{H} 3 \mathrm{~K} 27$, and $\mathrm{H} 4 \mathrm{~K} 20$. Methylation at $\mathrm{H} 3 \mathrm{~K} 9$ is implicated in the silencing of euchromatic genes as well as forming silent heterochromatin. Like lysine methylation, arginine methylation correlates with both permissive and non-permissive chromatin states. Enzymes (protein arginine methyltransferases, PRMT's) are recruited to promoters by transcription factors (24). Open chromatin and transcriptionally active regions are most commonly recognized by two different methylation status of H3K4, H3K4 di-methylation (H3K4me2) and tri-methylation (H3K4me3). H3$\mathrm{K} 4 \mathrm{me} 3$ is a key hallmark of active promoters. H3K4me2 marks both promoters and enhancers, and commonly occur at $\mathrm{CpG}$ islands. Genes associated with silenced DNA and heterochromatin are generally associated with methylations at lysine 9 or 27 of histone $\mathrm{H} 3$ (H3K9 and H3K27). Coexistence of repressing H3K27me3 and activating H3K4me3 epigenetic marks on same DNA region, usually at regulatory regions of gene promoters, are called 'bivalent domains'. This bivalency fine-tunes gene expression during development and contemporarily, keep gene expression in a poised or primed state that allows for either rapid activation or stable silencing upon differentiation of hematopoietic cells (Fig. 2) (25).

Chromatin-modifying polycomb group ( $\mathrm{PcG}$ ) proteins form large multimeric structuresdistinctly, polycomb repressive complex 1 and 2 (PRC1 and PRC2). The PRC1 includes BMI1, RING1/2, MEL-18, RAE28/MPH1, and M33/CBX2. PRC1 represses genes by mechanisms such as chromatin compaction and decreasing nucleosomal turnover. As observed in knockout mouse lines for Ring1B, Bmil, Rae28, and Mel18, mice harboring mutations in the components of PRC1 generate hematopoietic abnormalities. Once circumscribed to the chromatin, Ring1B and Bmil ubiquitinate histone H2A at lysine 119 (H2AK119ub) (26), which is considered to be involved in transcriptional repression by inhibiting transcription initiation or by restraining RNA polymerase II from elongation. It is not yet confirmed that whether PRCl function as a transcriptional repressor is dependent or independent of its H2Aub ligase catalytic activity (27). PRC2 stabilize repressive chromatin structure through the function of chromatin modifiers such as enhancer of zeste (EZh2), embryonic ectoderm development (Eed) protein and suppressor of zeste 12 (Suz12). PRC2 is responsible for di and tri-methylation of lysine 27 of histone $\mathrm{H} 3$ (H3K27me2/me3) to promote 
A

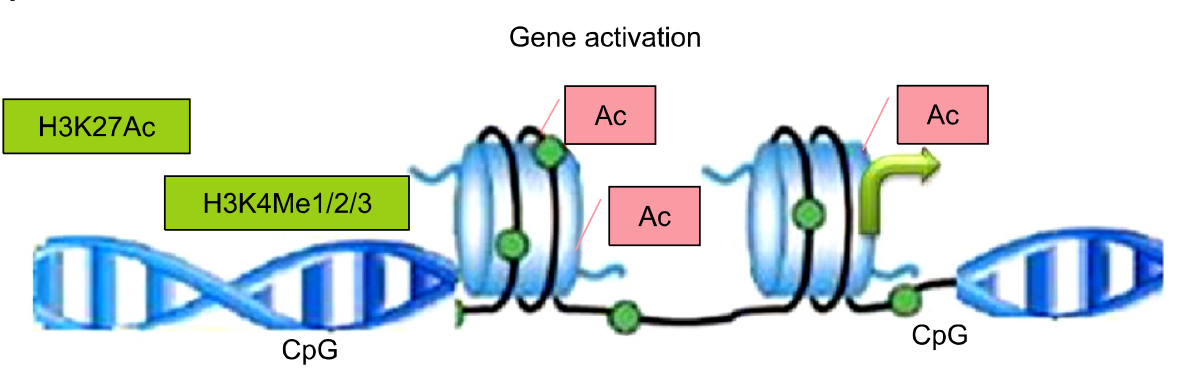

B

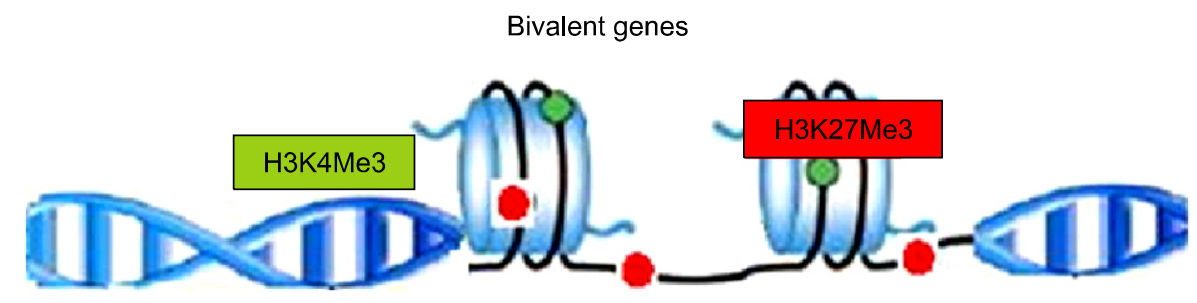

C

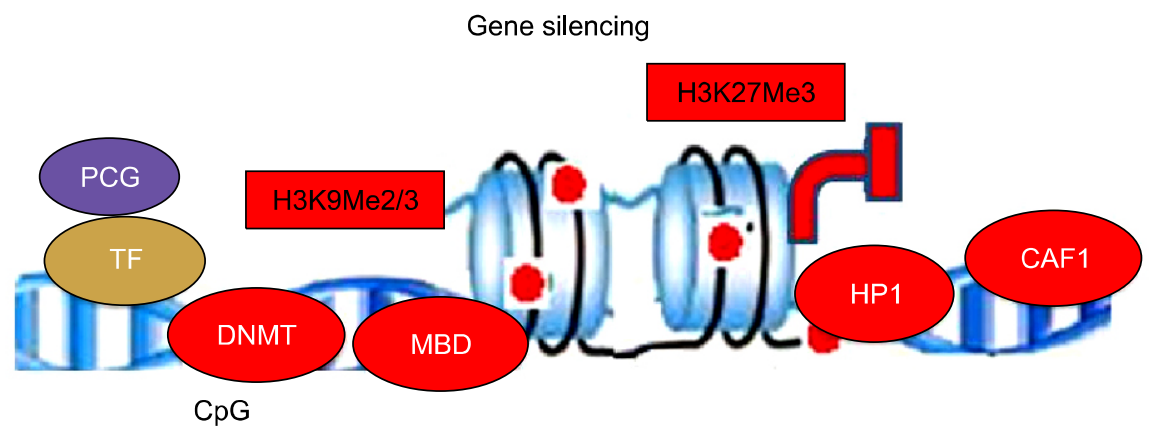

Fig. 2. Histone modifications: Active genes: Open chromatin structure of transcriptionally active gene with loosely spaced nucleosomes. Acetylation of lysine neutralizes the positive charge, reducing affinity between histone and DNA, which functions as platforms for the recruitment of transcription factors or chromatin remodelers, thus histone modifications directly effects nucleosomal architecture. H3K4me3 is enriched around transcription start sites. H3K4me1 is enriched around enhancers and downstream. H3K27ac is enriched around active enhancers and transcription start sites. Bivalent genes: In undifferentiated stem cells, both H3K4me3 and H3K27me3 (active and inactive marks, respectively) are enriched around transcription start sites on many genes. The multiple coexisting histone modifications are associated with activation and repression. Inactive genes: H3K9me3 is broadly distributed on inactive regions. H3K27me3 and H3K9me3 are usually not colocalized. Proteins associated for transcription silencing are DNMT- DNA methyltransferase, MBD-Methyl-binding domain, HP-1-Heterochromatin protein and CAF-1-Chromatin assembly factor1. transcriptional repression. HSCs express high levels of Bmil, a member of the PcG of transcription repressors. Furthermore, PRC1 component Bmil plays an important role in HSC self-renewal and lymphocyte differentiation by transcriptional repression of genes involved in cellular senescence (p16Ink4a, p19Arf, and p53) (28). Increased expression of the p53 target gene, Wig1 in Bmil ${ }^{-/-}$bone marrow suggests that p19Arf pathway gets activated in Bmildeficient hematopoietic cells (29). Bmil is critical in preventing Ikaros-mediated lymphoid priming, thus, prevents premature lineage specification of HSPCs (30). It has recently become clear that Bmil also functions in the protection against oxidative stress. Bmil is frequently overexpressed in human hematopoietic malignancies. Down modulation of Bmil impairs self-renewal and long-term expansion of leukemic stem cells. Bmil also functions in an orchestration of the symmetry of HSC divisions and bypassing senescence (31). Changes in the expression of individual PcG genes cause perturbations in the composition of the PRC, which affect overall PRC enzymatic activity. Overexpression of BMI1 is associated with malignant hematopoiesis (32). EZH2 over-expression direct stem cells toward a cancer stem cell state. EZH2 serve as a tumor-suppressor gene as itsinhibition in cancer stem cells offers a potential therapeutic approach to counter the aberrant activation in lymphoma and leukemia (33).

\section{Histone demethylation}

Histone demethylases (HDMs) reportedly reshape the epigenome of hematopoietic stem cell and thus regulate its fate. There are two families of HDMs, the amine oxidase enzyme, known as lysine demethylase (LSD1) and the Jumonji C (JmjC) domain-containing family. LSD1 forms a core complex with corepressors, including $\mathrm{HDACl} / 2$ and CoRESTand repress the transcription of target genes. LSD1 specifically removes one or two methyl groups from 
histone $\mathrm{H} 3$ lysine 4 (H3K4) or 9 (H3K9), depending on the cellular context and the presence of cofactors, thereby repressing or activating transcription. Overexpression of the shortest isoform of LSD1, which is specifically repressed in quiescent HSCs,demethylates histone H3K9 more efficiently than other isoforms $(34,35)$. LSD1 mediates erythroid differentiation via epigenetic modification of the GATA-2 locus and also increase self-renewal potential via upregulation of the HoxA family but retains differentiation ability towards T-cell lineages at transcriptome levels in HSCs. LSD1 has been identified as component of a transcription activation complex containing MLL1. The dysregulation of MLL in acute myeloid leukemia suggests that LSD1 may also have links to leukemogenesis. LSD1 overexpression seemingly is directly implicated for priming of HSCs towards malignant transformation and progression to T-cell leukemogenesis. Thus, provides a novel strategy for early diagnosis and effective treatment of the disease (36).

The second family of HDMs includes the JmjC enzymes, which catalyse the removal of mono-, di- and trimethyllysines. Several JmjC domain proteins have been identified with different specificities including JHDM1, JHDM2, JMJD2 and Jumonji AT-rich interactive domain 1 (JARID1). The interaction of the JmjC HDM JARID1d with the polycomb-like protein RING6a/MBLR showed that RING6a/MBLR enhance JARID1d-mediated H3K4 demethylation, associated with transcriptional repression. JHDM1b has also been shown to interact with PcG proteins in a complex containing Ringlb/Rnf2 and the Bcl6 interacting co-repressor among other proteins (37). This co-repressor complex may be implicated in gene repression mediated by Bcl6, a transcription factor frequently dysregulated in B-cell lymphomas. Deletion of JARID1b compromises HSC self-renewal capacity, which suggests that JARIDlb is a positive regulator of HSC potential (38). JARID1b/KDM5b is highly expressed in primitive hematopoietic compartments and is overexpressed in acute myeloid leukemias (5).

\section{Histone ubiquitination}

Among the histone octamer, $\mathrm{H} 2 \mathrm{~A}$ is the most abundant ubiquitinated protein and PRC1 is the main H2A ubiquitin (H2Aub) ligase. Ubiquitination of histone $\mathrm{H} 2 \mathrm{~A}$ $(\mathrm{H} 2 \mathrm{~A}-\mathrm{K} 119 \mathrm{u})$ is a common chromatin modification associated with gene silencing, and controlled by the activities of ubiquitin ligating and deubiquitinating enzymes. The RING1 subunit of PRC1 monoubiquitinylates histone $\mathrm{H} 2 \mathrm{~A}$ at lysine 119 (H2A-K119u), which promote transcrition repression and chromatin compaction. Another
H2A-deubiquitinating enzyme, Mysm1 plays a critical role in HSC maintenance, self-renewal, genetic stability in hematopoietic progenitors, and development of lymphoid and erythroid lineages $(39,40)$. Despite its important function and early discovery, histone ubiquitination remains the least understood compared with other histone modifications.

\section{Conclusions}

The cumulative research data demonstrate the crucial role of epigenetic players in stem cell self-renewal and differentiation gene expression patterns. The startling development of reprogramming technology has catapulted stem cell role in regenerative medicine and disease modeling and offers new hope for personalized regenerative cell therapies. Recently developed high-throughput genomic tools greatly facilitate the study of epigenetic regulation in pluripotent stem cells by mapping chromatin-nuclear matrix interactions. Elucidating epigenetic landscape will have important implications to expedite manipulation of stem and progenitor cells for regenerative applications and would render novel targets to tackle human diseases prospectively, leading to new therapeutic avenues in regenerative medicine.

\section{Acknowledgments}

We are thankful to Dr R.P. Tripathi, Director, Institute of Nuclear Medicine and Allied Sciences, Brig. S.K. Mazumdar Marg, Timarpur, Delhi-110054 for providing us necessary facilities and support. Authors also thank the Ministry of Defence, Department of Defence Research and Development for research grant. Shilpa Sharma in particular thanks Indian Council of Medical Research (ICMR) for the award of Research Associateship.

\section{Potential competing interests}

The authors have no conflicting financial interest.

\section{References}

1. Sharma S, Gurudutta GU, Satija NK, Pati S, Afrin F, Gupta P, Verma YK, Singh VK, Tripathi RP. Stem cell c-KIT and HOXB4 genes: critical roles and mechanisms in self-renewal, proliferation, and differentiation. Stem Cells Dev 2006;15:755-778

2. Zhou Y, Kim J, Yuan X, Braun T. Epigenetic modifications of stem cells: a paradigm for the control of cardiac progenitor cells. Circ Res 2011;109:1067-1081

3. Bottardi S, Ghiam AF, Bergeron F, Milot E. Lineage-specific transcription factors in multipotent hematopoietic 
progenitors: a little bit goes a long way. Cell Cycle 2007; 6:1035-1039

4. Klose RJ, Zhang Y. Regulation of histone methylation by demethylimination and demethylation. Nat Rev Mol Cell Biol 2007;8:307-318

5. Rice KL, Hormaeche I, Licht JD. Epigenetic regulation of normal and malignant hematopoiesis. Oncogene 2007;26: 6697-6714

6. Cedar H, Bergman Y. Linking DNA methylation and histone modification: patterns and paradigms. Nat Rev Genet 2009;10:295-304

7. Branco MR, Ficz G, Reik W. Uncovering the role of 5-hydroxymethylcytosine in the epigenome. Nat Rev Genet 2011;13:7-13

8. Calvanese V, Fernández AF, Urdinguio RG, Suárez-Alvarez B, Mangas C, Pérez-García V, Bueno C, Montes R, Ramos-Mejía V, Martínez-Camblor P, Ferrero C, Assenov Y, Bock C, Menendez P, Carrera AC, Lopez-Larrea C, Fraga MF. A promoter DNA demethylation landscape of human hematopoietic differentiation. Nucleic Acids Res 2012;40:116-131

9. Trowbridge JJ, Orkin SH. Dnmt3a silences hematopoietic stem cell self-renewal. Nat Genet 2011;44:13-14

10. Challen GA, Sun D, Mayle A, Jeong M, Luo M, Rodriguez B, Mallaney C, Celik H, Yang L, Xia Z, Cullen S, Berg J, Zheng Y, Darlington GJ, Li W, Goodell MA. Dnmt3a and Dnmt3b have overlapping and distinct functions in hematopoietic stem cells. Cell Stem Cell 2014;15:350-364

11. Trowbridge JJ, Snow JW, Kim J, Orkin SH. DNA methyltransferase 1 is essential for and uniquely regulates hematopoietic stem and progenitor cells. Cell Stem Cell 2009; 5:442-449

12. Rose NR, Klose RJ. Understanding the relationship between DNA methylation and histone lysine methylation. Biochim Biophys Acta 2014;1839:1362-1372

13. Vasanthakumar A, Zullow H, Lepore JB, Thomas K, Young N, Anastasi J, Reardon CA, Godley LA. Epigenetic Control of Apolipoprotein E Expression Mediates Gender-Specific Hematopoietic Regulation. Stem Cells 2015 doi: 10.1002/ stem.2214. [Epub ahead of print]

14. Yang J, Corsello TR, Ma Y. Stem cell gene SALL4 suppresses transcription through recruitment of DNA methyltransferases. J Biol Chem 2012;287:1996-2005

15. Gao C, Kong NR, Chai L. The role of stem cell factor SALL4 in leukemogenesis. Crit Rev Oncog 2011;16:117-127

16. Di Croce L, Raker VA, Corsaro M, Fazi F, Fanelli M, Faretta M, Fuks F, Lo Coco F, Kouzarides T, Nervi C, Minucci S, Pelicci PG. Methyltransferase recruitment and DNA hypermethylation of target promoters by an oncogenic transcription factor. Science 2002;295:1079-1082

17. Santini V, Melnick A, Maciejewski JP, Duprez E, Nervi C, Cocco L, Ford KG, Mufti G. Epigenetics in focus: pathogenesis of myelodysplastic syndromes and the role of hypomethylating agents. Crit Rev Oncol Hematol 2013;88: 231-245

18. Sun XJ, Man N, Tan Y, Nimer SD, Wang L. The role of histone acetyltransferases in normal and malignant hematopoiesis. Front Oncol 2015;5:108

19. Muñoz P, Iliou MS, Esteller M. Epigenetic alterations involved in cancer stem cell reprogramming. Mol Oncol 2012;6:620-636

20. Bug G, Gül H, Schwarz K, Pfeifer H, Kampfmann M, Zheng X, Beissert T, Boehrer S, Hoelzer D, Ottmann OG, Ruthardt M. Valproic acid stimulates proliferation and self-renewal of hematopoietic stem cells. Cancer Res 2005;65:2537-2541

21. Walasek MA, Bystrykh L, van den Boom V, Olthof S, Ausema A, Ritsema M, Huls G, de Haan G, van Os R. The combination of valproic acid and lithium delays hematopoietic stem/progenitor cell differentiation. Blood 2012;119:3050-3059

22. Sharma S, Kelly TK, Jones PA. Epigenetics in cancer. Carcinogenesis 2010;31:27-36.

23. Vaissière T, Sawan C, Herceg Z. Epigenetic interplay between histone modifications and DNA methylation in gene silencing. Mutat Res 2008;659:40-48

24. Kouzarides T. Chromatin modifications and their function. Cell 2007;128:693-705

25. Voigt P, Tee WW, Reinberg D. A double take on bivalent promoters. Genes Dev 2013;27:1318-1338

26. Eskeland R, Leeb M, Grimes GR, Kress C, Boyle S, Sproul D, Gilbert N, Fan Y, Skoultchi AI, Wutz A, Bickmore WA. Ring1B compacts chromatin structure and represses gene expression independent of histone ubiquitination. Mol Cell 2010;38:452-464

27. Valk-Lingbeek ME, Bruggeman SW, van Lohuizen $M$. Stem cells and cancer; the polycomb connection. Cell 2004; 118:409-418

28. Iwama A, Oguro H, Negishi M, Kato Y, Nakauchia H. Epigenetic regulation of hematopoietic stem cell self-renewal by polycomb group genes. Int J Hematol 2005; 81:294-300

29. Park IK, Qian D, Kiel M, Becker MW, Pihalja $M$, Weissman IL, Morrison SJ, Clarke MF. Bmi-1 is required for maintenance of adult self-renewing haematopoietic stem cells. Nature 2003;423:302-305

30. Arranz L, Herrera-Merchan A, Ligos JM, de Molina A, Dominguez O, Gonzalez S. Bmil is critical to prevent Ikaros-mediated lymphoid priming in hematopoietic stem cells. Cell Cycle 2012;11:65-78

31. Schuringa JJ, Vellenga E. Role of the polycomb group gene BMI1 in normal and leukemic hematopoietic stem and progenitor cells. Curr Opin Hematol 2010;17:294-299

32. Lessard J, Sauvageau G. Bmi-1 determines the proliferative capacity of normal and leukaemic stem cells. Nature 2003; 423:255-260

33. Lund $\mathrm{K}$, Adams PD, Copland M. EZH2 in normal and malignant hematopoiesis. Leukemia 2014;28:44-49

34. Wada T, Koyama D, Kikuchi J, Honda H, Furukawa Y. Overexpression of the shortest isoform of histone demethylase LSD1 primes hematopoietic stem cells for malignant transformation. Blood 2015;125:3731-3746 
35. Forneris F, Binda C, Dall'Aglio A, Fraaije MW, Battaglioli E, Mattevi A. A highly specific mechanism of histone H3-K4 recognition by histone demethylase LSD1. J Biol Chem 2006;281:35289-35295

36. Guo Y, Fu X, Jin Y, Sun J, Liu Y, Huo B, Li X, Hu X. Histone demethylase LSD1-mediated repression of GATA-2 is critical for erythroid differentiation. Drug Des Devel Ther 2015;9:3153-3162

37. Sánchez C, Sánchez I, Demmers JA, Rodriguez P, Strouboulis J, Vidal M. Proteomics analysis of Ring1B/Rnf2 interactors identifies a novel complex with the Fbxl10/ Jhdm1B histone demethylase and the Bcl6 interacting corepressor. Mol Cell Proteomics 2007;6:820-834
38. Stewart MH, Albert M, Sroczynska P, Cruickshank VA, Guo Y, Rossi DJ, Helin K, Enver T. The histone demethylase Jaridlb is required for hematopoietic stem cell self-renewal in mice. Blood 2015;125:2075-2078

39. Cao R, Tsukada Y, Zhang Y. Role of Bmi-1 and Ring1A in H2A ubiquitylation and Hox gene silencing. Mol Cell 2005;20:845-854

40. Gatzka M, Tasdogan A, Hainzl A, Allies G, Maity P, Wilms C, Wlaschek M, Scharffetter-Kochanek K. Interplay of H2A deubiquitinase 2A-DUB/Mysml and the p19(ARF)/p53 axis in hematopoiesis, early T-cell development and tissue differentiation. Cell Death Differ 2015;22:1451-1462 